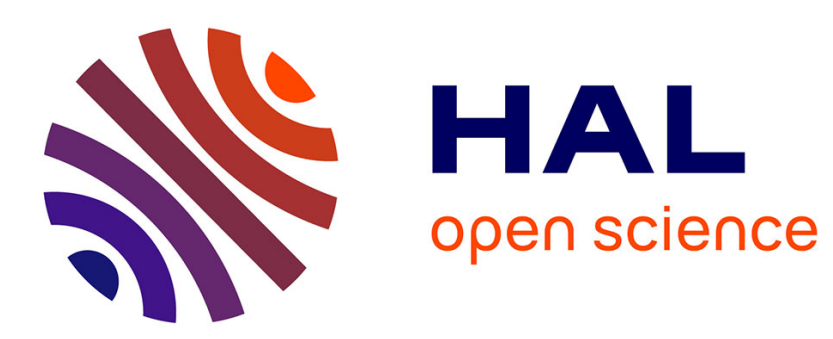

\title{
Experimental evidence of ground albedo neutron impact on Soft Error Rate for nanoscale devices
}

\author{
Guillaume Hubert, Laurent Artola
}

\section{To cite this version:}

Guillaume Hubert, Laurent Artola. Experimental evidence of ground albedo neutron impact on Soft Error Rate for nanoscale devices. IEEE Transactions on Nuclear Science, 2018, 66 (1), pp.1-8. 10.1109/TNS.2018.2877263 . hal-02301042

\section{HAL Id: hal-02301042 \\ https://hal.science/hal-02301042}

Submitted on 30 Sep 2019

HAL is a multi-disciplinary open access archive for the deposit and dissemination of scientific research documents, whether they are published or not. The documents may come from teaching and research institutions in France or abroad, or from public or private research centers.
L'archive ouverte pluridisciplinaire HAL, est destinée au dépôt et à la diffusion de documents scientifiques de niveau recherche, publiés ou non, émanant des établissements d'enseignement et de recherche français ou étrangers, des laboratoires publics ou privés. 


\title{
Experimental evidence of ground albedo neutron impact on Soft Error Rate for nanoscale devices
}

\author{
G. Hubert, L. Artola
}

\begin{abstract}
This work demonstrates the experimental evidence of ground albedo neutron impact on Soft Error Rate (SER) for nanoscale devices. The SER of a $45 \mathrm{~nm}$ technology was measured according to an experimental protocol using a californium sealed source and several scenes based on material blocks. High density polyethylene and concrete materials were considered to investigate the intrinsic role in albedo neutron productions and their effect on devices. Results show the impact in the spectrum concern mainly energies below $5 \mathrm{MeV}$. Devices are characterized by a sensitivity which varies according to the presence or not of thethe material block. Simulations using GEANT4 and MUSCA SEP3 tool were performed to extend analyses. A final part is devoted to investigate the impact of ground albedo neutrons on SER by considering realistic terrestrial neutron field.
\end{abstract}

Index Terms - Ground albedo neutron, Soft Error Rate (SER), spectrometry, MUSCA SEP3, SEE tests

\section{INTRODUCTION}

$S$ INGLE Event Effects (SEE) risks are a growing concern for avionic and terrestrial applications [1][2]. Thus, the atmospheric radiation characterization is essential to assess their effects on electronic systems.

Primary galactic and solar cosmic-rays (GCR and SCR, respectively) travel toward the Earth at a rate which varies according to the solar activity cycle (11-year) and solar events which can considerably increase particle fluxes over durations of few hours (extreme event as Ground Level enhancement). The shielding of primary particles, provided by the geomagnetic field, is described by the cutoff rigidity and is defined as the minimum momentum per unit charge that an incident particle can have and still reach a given location above the earth. The cutoff rigidity is greatest at the geomagnetic equator and smallest at high latitudes. In the atmosphere, primary Cosmic Rays (CR) interact with the nuclei of atmospheric gases, creating cascades of secondary particles including neutrons, protons, pions, muons and electrons. These secondary particles are named secondary Cosmic Rays.

Secondary CRs are also attenuated as they interact with nuclei in the Earth's atmosphere, and the number of interactions and the intensity of cosmic-ray particles decrease with increasing atmospheric depth [3][4]. High-energy neutrons (typically $>10 \mathrm{MeV}$ ) colliding with nuclei can produce fast evaporation neutrons, i.e. on the order of $1 \mathrm{MeV}$. Evaporation neutrons are reduced to epithermal and thermal

G. Hubert and L. Artola are with ONERA DPHY, University of Toulouse, 31055 Toulouse, France (e-mail: firstname.name@onera.fr). neutrons as they travel and interact with the elements of the air, vegetation, and soil [5][6]. Hydrogen is by far the most important element for the moderation (loss of energy) of evaporation and epithermal neutrons as both the cross-section and the energy loss per collision is relatively high. Indeed, the intensity of neutrons produced by $\mathrm{CR}$ is sensitive to soil moisture in the upper decimeters of the ground. The interactions of neutrons with the soil induce re-emission of particles named albedo. This contribution is therefore additional to neutrons from the cosmic shower.

Previous works [7][8] investigated the impact of ground albedo neutrons in spectrum, particularly in the thermal, epithermal and evaporation domains. The objective was to interpret the neutron fluxes dynamics recorded in high altitude stations (in the Pic-du-Midi in French Pyrenees and the Concordia polar station) and to correlate these variations according to the hydrogen content mainly induced by precipitations and snowfalls. Transport simulation results were compared with measured spectra (Bonner sphere system neutron spectrometer) in the Concordia (Antarctic) and the Pic-du-Midi (France) stations. Thus, the evaporation domain, which implies neutrons with energy between $0.2 \mathrm{eV}$ to 20 $\mathrm{MeV}$, is a field of interest since these energies are likely to induce SEEs in electronics, particularly for advanced technologies. Indeed, most recent SEE tests conducted in ground facilities show threshold neutron energies in the order of $\mathrm{MeV}$. First investigations devoted to impacts of groundalbedo neutron on SER assessment were presented in [7]. Contributions of ground albedo neutrons on the SER risk were investigated using Monte-Carlo simulations and MUSCA SEP3 [9]. Results have showed that evaporation neutrons contribute significantly to SER, around 20 to $40 \%$ and up to $50 \%$ considering typical critical charges and low critical charges, respectively.

This work proposes to demonstrate experimentally the impact of ground albedo neutrons on the SER considering a commercial FPGA (Feld Programmable Gate Array) SRAM based (Static Random Access Memory) designed in 45-nm. Thus, the SER of SPARTAN6 FPGA was measured according to an experimental protocol using a californium source and material blocks to simulate the soil impact. Two materials are selected, i.e. high density polyethylene (HDPE) and concrete. SER are compared with SER issued from reference configuration without soil. The final part is devoted to investigate the impact of ground albedo neutrons on SER by considering a realistic terrestrial neutron field. 


\section{EXPERIMENTAL AND MODELING PLATFORMS}

\section{A. Experimental platform based on SEE tester and neutron spectrometer}

\section{1) Global approach}

To evaluate the impact of albedo neutrons on the SEE rate, neutron irradiations were performed using a ${ }^{252} \mathrm{Cf}$ source in the ONERA's facility, called CIRIL. A HDPE block (chemical formula: $\left(\mathrm{C}_{2} \mathrm{H}_{4}\right)_{\mathrm{n}}$, density $\left.=0.941 \mathrm{~g} / \mathrm{m}^{3}\right)$ and a concrete (density $=2.3 \mathrm{~g} / \mathrm{m}^{3}$ ) block were used.

The concrete materials are a mixture of elements with relative proportions that vary according to types and specificities. Thus, the classical cement is mainly composed by oxides as lime $(\mathrm{CaO})$, silica $\left(\mathrm{SiO}_{2}\right)$, alumina $\left(\mathrm{Al}_{2} \mathrm{O}_{3}\right)$, and iron $\left(\mathrm{Fe}_{2} \mathrm{O} 3\right)$. Depending on the properties, there are also magnesia, alkalizes and sulfuric anhydrite. Moreover, the sand mainly contains silica. In this study, the classical concrete material is considered [10], and contains the chemical elements with the following proportions (in \%): $\mathrm{H}(0.94), \mathrm{C}$ (0.09), O (53.66), Na (0.46), Mg (0.12), Al (1.32), Si (36.74), $\mathrm{S}(0.08), \mathrm{K}(0.31), \mathrm{Ca}(5.65)$ and $\mathrm{Fe}(0.63)$. In order to conduct coupled experimental and simulation investigations, three complementary approaches are proposed:

1) Radiation field measurements based on a neutron spectrometer to record the neutron spectra as function of the scene characteristics,

2) SER measurements based on SEE test board to correlate the scene characteristics and the observed variations in failure rate,

3) SEE modelling using spectra measurements and device description (topology and SEU occurrence) to correlate both experimental approaches.

Fig. 1(a) details the experimental facility. The neutron facility is based on ${ }^{252} \mathrm{Cf}$ sealed source which characterized by spontaneous fission yielding an average of 3.75 fast neutrons per fission. The source produces $2.3 .10^{9}$ neutron. $\mathrm{s}^{-1} \cdot \mathrm{mg}^{-1}$ and the associated yield is thus 0.116 neutron $\mathrm{s}^{-1} \cdot \mathrm{Bq}^{-1}$. The energy spectrum of ${ }^{252} \mathrm{Cf}$ source, being a fission spectrum, is well characterized and can be described by Maxwellian distribution. The material block was located behind the measuring system which can be the neutron spectrometer (b) or the SEE test board (c). In both cases, the presence or absence of the block will make it possible to evaluate their effect, i.e. the contribution of albedo neutrons can be deduced by differentiating measurements. Moreover, it is possible to compare the impact of the material properties in comparing HDPE and concrete results.

The source to FPGA distance is $5 \mathrm{~mm}$ while the distance between the blocks and the FPGA board is $1 \mathrm{~cm}$. The objective was to maximize the albedo neutron contribution to spectra and SEE occurrence. The HDPE and concrete blocks are composed by elementary bricks and their total thicknesses are $30 \mathrm{~cm}$ (this thickness being limited by the facility dimensions.

\section{2) Neutron spectrometer}

The neutron spectrometer used in this work [1][11] is similar to instruments operated in the Pic-du-Midi observatory and in the Concordia station (Antarctica) [12], and devoted to characterize the high-altitude neutron spectra. This instrument was therefore presented in previous papers [1][11], and its description is limited to their main characteristics. It is based on multisphere system using ten high density polyethylene homogeneous spheres with increasing diameters (from 3" to 12"). Two additionnal spheres integrate inner tungsten and lead shells to increase their response to fast neutrons (typically higher than $20 \mathrm{MeV}$, i.e. cascade neutrons). Considering the typical energies induced by the californium source, metallic spheres are not required in measurements. The neutron detection is based on ${ }^{3} \mathrm{He}$ spherical proportional counter placed in the center of each HDPE sphere, and allows the thermal neutron detection thanks to the ${ }^{3} \mathrm{He}(\mathrm{n}, \mathrm{p})^{3} \mathrm{H}$ reaction. The thickness of the spheres aims to thermalize the energetic neutron by elastic collisions with the hydrogen nuclei. This detection principle is named "Bonner sphere".

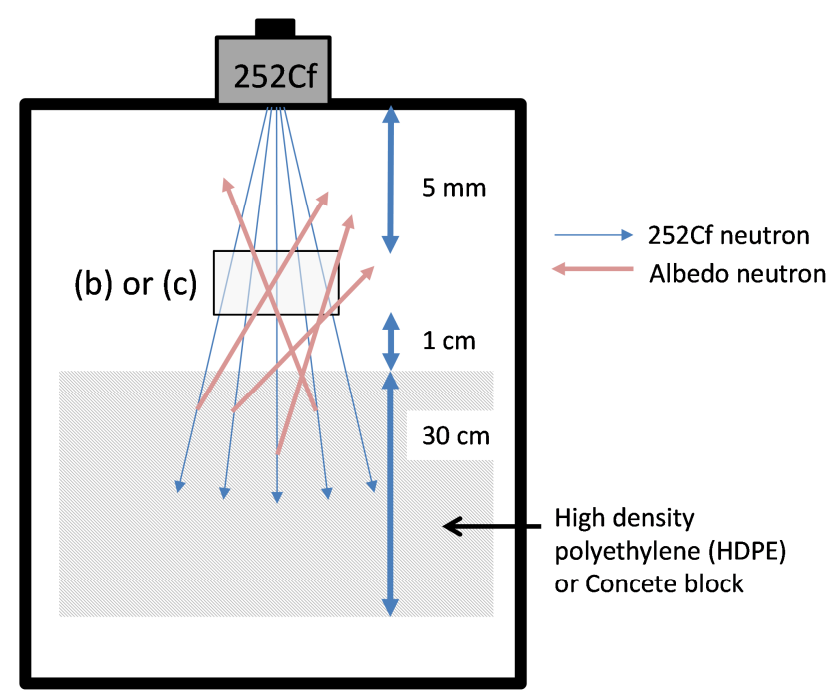

(a) Experimental facility

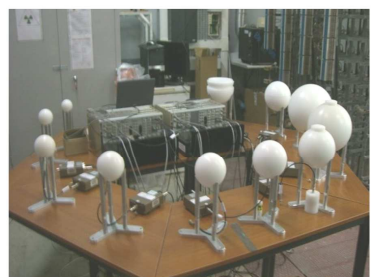

(b) Neutron spectrometer

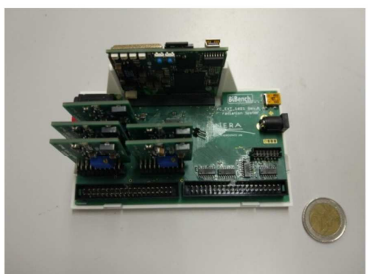

(c) SEE test board
Fig. 1 Overview of the global approach, (a) the description of the experimental facility (b) a pictures of the neutron spectrometer (c) and the SEE test board.

When spheres are exposed to a neutron field characterized by a spectral fluence $\phi_{E}(E)$ (in $\mathrm{cm}^{-2} \cdot \mathrm{MeV}^{-1}$ ), each detector integrates a count number or a spectrum depending on whether counter chains or analyzer multi-channels are used, respectively. Thus, a set of readings $M_{i}$ is collected for each integration time, generally chosen in the range of a few minutes when the atmospheric environment is studied, but in the order of a few seconds when an artificial field is recorded. To deduce the neutron spectrum from the count rates, the fluence response functions of each detector $R_{i}(E)$ were calculated using nuclear transport code (MCNPx, GEANT4) 
and by solving the system of equations. There are several referent tools that can solve this system of equation, among which the GRAVEL unfolding code [13]. Since the number of detections (issued from 10 spheres) is insufficient in comparison to the neutron spectrum range, it is necessary to define a priori information and it requires an estimation of the shape of the neutron spectrum at the measurement location [13][14].

In the case of atmospheric neutron field, measurements are performed simultaneously on each sphere, under the pretext that the radiation field is negligibly affected by the positioning of detectors on the allocated area (including interaction between adjacent spheres). In this study devoted to characterize the field undergone by the DUT (Device Under Test), measurements are performed using each detector alternately. This approach is justified because the neutron field is stable.

\section{3) SEE test board and irradiated device}

ONERA developed a SEE test board, called BIBURAD, which allows for testing SRAM memories ship and embedded in FPGA. As mentioned, in this work, a SPARTAN 6 FPGA designed in 45-nm technology by Xilinx Series was tested. The reference is XC6SLX100.

The test board is composed by a mother board driven by a FPGA as shown in Fig. 1(c). This main board controls the functional behavior of the DUT located on a daughter board. For reliability reasons, no active component is on the daughter board excluding the DUT. During the irradiation runs, the test board reads the Bacl-RAM (BRAM) and the Configuration RAM (CRAM) blocks of the studied FPGA to detect potential SEUs. If an upset is detected, the test board reads again the memory block in order to confirm the occurrence of a SEU or to identify a Single Event Transient. Moreover, in order to prevent and detect Single Event Latchup, the power supply current is monitored. If the current increase over a determined threshold current, a SEL is considered and a off/on cycle is done on the DUT board.

\section{B. Test campaign details}

As presented in Fig. 2, measurements are conducted from May 2017 to June 2018. The irradiation steps require a significant duration to obtain a statistically relevant number of SEU. Preliminarily measurements were conducted until August 2017 to quantify the intrinsic daily SEU rate (without neutron source), including contributions induced by noises, Cosmic Rays or $\alpha$-SER. This rate is systematically taken into account in the analysis.

Thus, there are four steps for SEE measurements, including references (without material block) and configurations using HDPE or concrete blocks. Steps devoted to neutron field characterizations are time-consuming because each detector is alternately used. The cumulative irradiation time required for this study was in the order of 7 months.

\section{SEE simulations using MUSCA SEP3}

The SEE modeling is based on MUSCA SEP3 methodology presented in previous works [1][2][9]. The Monte Carlo method consists in considering a large number of random configurations (Monte-Carlo) for which a sequential modeling of each physical layer will be conducted, i.e. the interaction mechanisms, the transport in semiconductor and the electrical disturbance of the circuit. Each of these physical layers is based on reduced physical models or databases. This approach allows for considering the device description (layout, thickness details), its local and global environment (overlayer, structures) and the radiation field environment (nature, direction, spectrum and dynamics).

The ${ }^{252} \mathrm{Cf}$ source was modeled with the isotropic emission and energetic neutron spectra deduced from measurements (see Fig. 1). The interaction transport and the carrier deposition in active Silicon regions are based on databases containing secondary details induced by neutron silicon nuclear interactions for neutron energy range from low to 20 $\mathrm{MeV}$. Coulombian databases allow to quantify the energy loss of the secondary ions in matter and their 3D morphology deposits in the active areas [15]. This refinement is necessary given the level of integration of the studied technology. The transport/collection models and the occurrence criteria make it possible to calculate the collected charge and the impact of this charge level on the elementary memory cell.

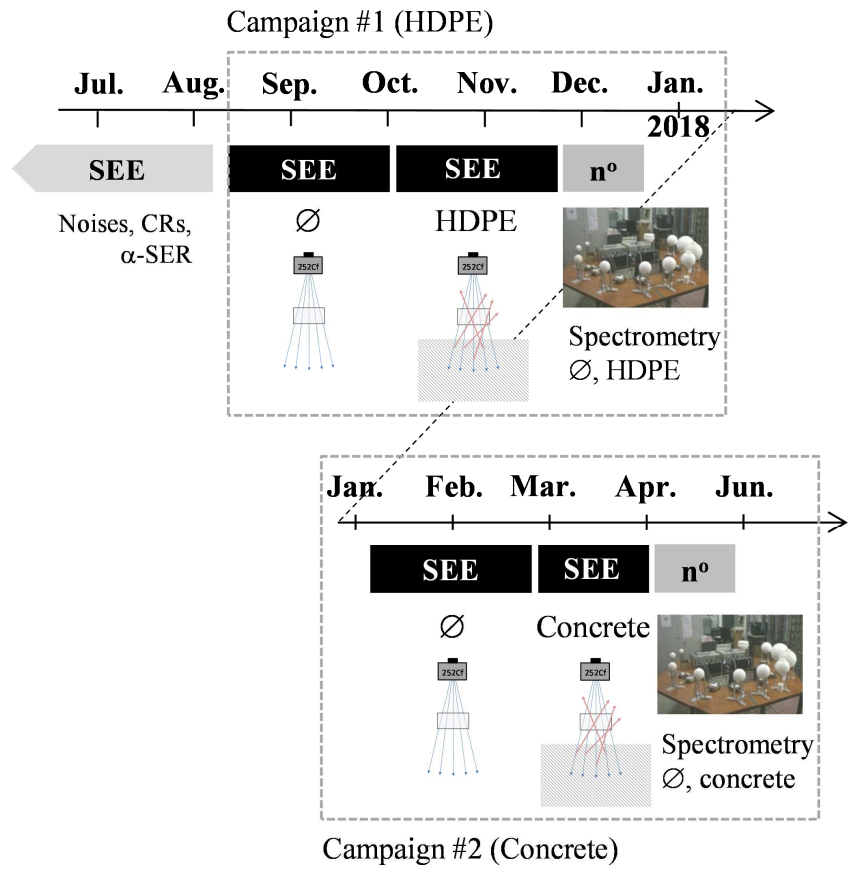

Fig. 2 Test campaign chronology including SEE rate measurements and neutron spectrometry characterization with and without material block. It is possible to distinguish the both test campaigns i.e. HDPE (\#1) and concrete (\#2), respectively.

\section{RESULTS AND ANALYSES}

\section{A. Measured and simulated Neutron spectra analyses}

Fig. 3 presents the neutron spectra measured without material block, using HDPE block and using concrete block placed behind the spectrometer. Enrichment of neutron populations below $5 \mathrm{MeV}$ is observed when the material block is placed behind the sphere. However, the HDPE induces a much larger enrichment in the evaporation domain. These differences are due to several factors, primarily the chemical 
composition, the density and the hydrogen content.

In order to quantify these variations, Fig. 4 presents the ratio between spectra deduced from experiment and calculations. Calculations are based on GEANT4 simulations reproducing the scene, i.e. geometries, the ${ }^{252} \mathrm{Cf}$ source and the HDPE block. Results show a satisfactory correlation between measurements and calculations. Deviations observed for low energies can be explained by the impact of the Bonner spheres, composed in HDPE, and not taken into account in the simulations. Indeed, this matter can induce additional albedo contributions. This hypothesis is reinforced by the fact that nuclear interaction models are quite validated (including response functions used for the deconvolution process).

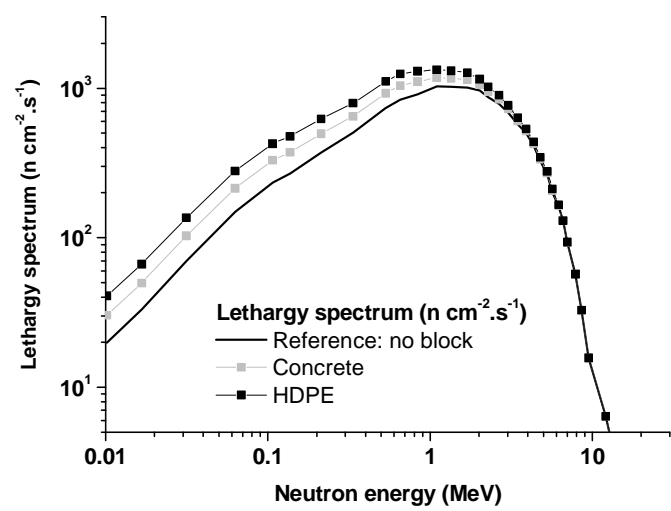

Fig. 3 Neutron spectra measured without material block, with a HDPE block and with a concrete block.

Results based on neutron spectrometry show an impact in the neutron spectrum for energies below to $5 \mathrm{MeV}$. It has been shown that nanoscale technologies can be sensitive to SEE for these energy ranges [16]. This suggests that technologies should have a sensitivity which varies according to the albedo neutron quantities, i.e. to the material and their properties.

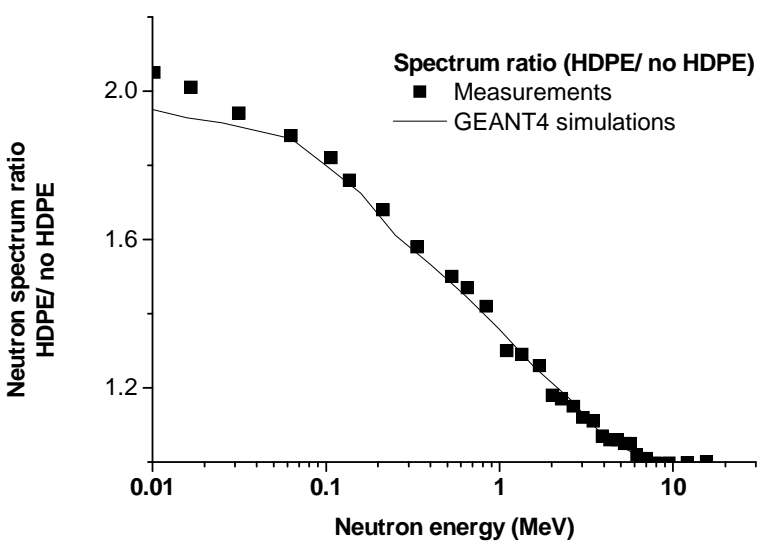

Fig. 4 Ratio HDPE/no HDPE as function of neutron energy (measurements and GEANT4 simulations).

\section{B. Impact of albedo neutrons on SEE rates}

First analyses demonstrate the significant impact of material block. To evaluate the impact on SER, the DUT was irradiated alternately using and not using a material block to simulate the soil. Fig. 5 presents the daily SER obtained considering HDPE block, including error bars defined by the cumulative events. At first the ${ }^{252} \mathrm{Cf}$ sealed source is placed in front of the DUT without material (behind).The test campaign is performed over a period which is long enough to reduce uncertainties, i.e. to impact of material block. Significant number of SEUs was detected after 45 days of irradiation then the HDPE block is introduced (behind the FPGA) and the SEU measurement was reseted. As shown in Fig. 5, an increase in the SER of 30\% was observed for the configuration using HDPE. In term of average SER, the presence of material block induced an increase of $2.8 \mathrm{SEU}$ per day. This trend is quite relevant with the observations devoted to spectrum analyses.

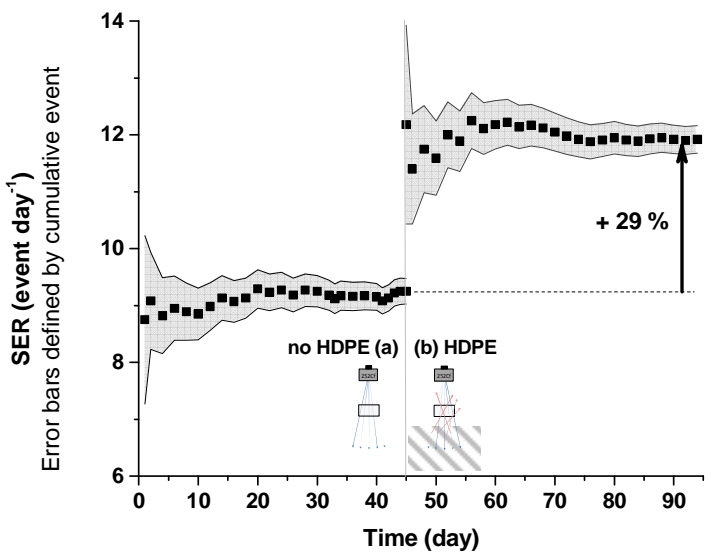

Fig. 5 Daily SER for each irradiation runs, (a) without and (b) with HDPE block behind the FPGA device. Error bars are calculated with cumulative events.

A second campaign using a concrete block was conducted. Reference measurements obtained without block are quite reproducible. Thus, after 40 days, the average SER issued from the first and the second campaign are of $9.19 \pm 0.22$ and $9.15 \pm 0.23 \mathrm{SEU} \mathrm{day}^{-1}$, respectively. Fig. 6 presents the average daily SER obtained using and not using the concrete block. The presence of the concrete block induces an increase of the SER in the order of $16 \%$. In term of average SER, the presence of material block induced an increase of $1.46 \mathrm{SEU}$ per day.

Table I summarizes the average SER measured during both campaigns, allowing to cross-compare the impact of material blocks. Time integrations are 40 and 30 days for configurations without and with block, respectively. Results indicate that the SER is dependent on the material, and this can be linked to the neutron spectrum variations. The magnitude of the soil influence depends on the technology through the failure threshold.

Simulations were performed using MUSCA SEP3. The material environment (facility, block ...), the neutron source and the SRAM were considered. The neutron source was described as a point source, isotropic and its spectrum is issued from measurements $\left({ }^{252} \mathrm{Cf}\right.$ source). The scene was described alternatively with and without material block. The technological critical charge value is not precisely known. A 
range of critical charge has been considered in order to get brief roadmap overview of albedo neutron contributions on the CMOS (Complementary Metal Oxide Semiconductor) integration scale. The impact of albedo is represented by the increase in the SER values obtained with material block.

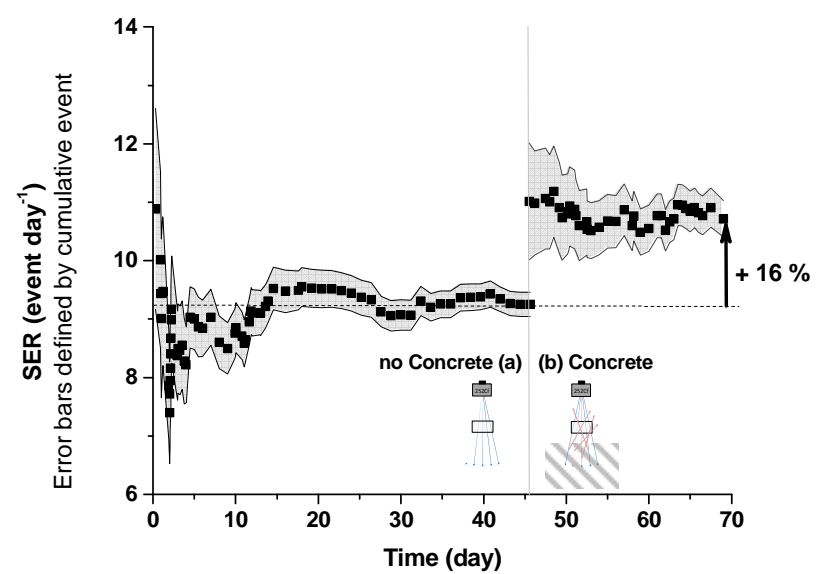

Fig. 6 Daily SER for each irradiation runs, (a) without and (b) with Concrete block behind the FPGA device. Error bars are calculated with cumulative events.

TABLE I. AVERAGE SER (IN SEU PER DAY) MEASURED DURING THE BOTH CAMPAIGN (USING HDPE AND CONCRETE, RESPECTIVELY), USING AND WITHOUT MATERIAL BLOCK

\begin{tabular}{lll}
\hline & Without material & With material \\
\hline Time integration (days) & 40 & 30 \\
\hline Campaign 1 (HDPE) & $9.19 \pm 0.22$ & $11.92 \pm 0.28$ \\
Campaign 2 (Concrete) & $9.15 \pm 0.23$ & $10.61 \pm 0.31$ \\
\hline
\end{tabular}

Fig. 7 shows the SER increase in percentage obtained by simulations, the neutron spectra corresponds to the measured neutron spectra presented in Fig. 3. Results show that albedo neutrons contribute significantly to SER, around 25 to $35 \%$ considering the typical critical charge value. The perimeter added in Fig. 7 corresponds to the relevant area for which both representative of typical critical charge values for the 45-nm technological node [2][17][19], and secondly orders of quantities deduced from SER measurements. Simulations are consistent with this perimeter, which makes it possible to correlate physically observed SER variations with albedos induced by the HDPE block.

There is no difference between the SER obtained with and without HDPE block for critical charge values up to $5 \mathrm{fC}$. Indeed, secondary particles or recoils do not generate enough carriers or charge in the sensitive zones. The event multiplicity can be easily extracted from simulations, and no event was observed. For energies typically less than few $\mathrm{MeV}$, the predominant mechanism is the elastic scattering inducing lowenergy silicon recoils. Thus, ranges are very short and LET values are conversely high (i.e. Bragg Peak), which is not favorable from the point of view of the multiple event occurrence. Results show that albedo neutrons are mainly characterized by energies below $5 \mathrm{MeV}$, i.e. energy range of evaporation neutrons in the atmospheric fields. Hydrogen content in the soil (wet or liquid water thickness) will impact the evaporation ground spectra and given the SEU sensitivity of nanoscale devices, it is necessary to extend spectrum specifications for SER calculations. It also implies the need to describe soil properties (i.e. composition, moisture etc.).

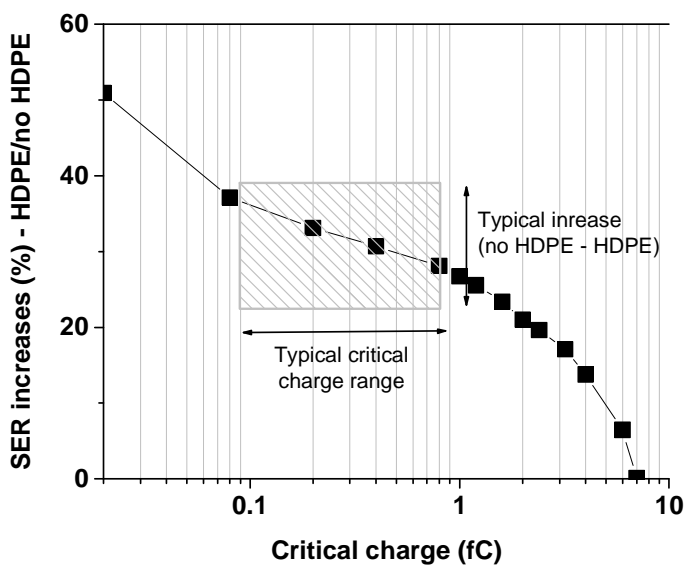

Fig. 7 SER increase between HDPE and no HDPE configurations as function of the critical charge.

\section{REALISTIC TERRESTRIAL ENVIRONMENT}

The energy range of the ${ }^{252} \mathrm{Cf}$ source is suitable for studying the impact of neutron albedo. However, the purpose is also to take into account this impact in the SEU risk assessment for realistic terrestrial spectra. Previous works [8] considering terrestrial environment had shown that soil properties impact the ground-albedo neutron production and SERs (only based on simulations). Roles of the porosity and the water content were investigated.

Analyzes consist in discriminating the contributions of the neutron spectrum resulting from the atmospheric shower and the albedo neutrons induced by the soil. The calculations were obtained by considering the Paris city location (i.e. $48^{\circ} 51^{\prime} 12^{\prime \prime}$ $\mathrm{N}, 2^{\circ} 20^{\prime} 55^{\prime \prime} \mathrm{E}$ and $+42 \mathrm{~m}$ ) and were based on ATMORAD [20]-[12]. The Solar activity corresponds to the June 2018 period, i.e. a quiet activity characterized by a solar modulation of $600 \mathrm{MV}$. Validations of ATMORAD [20] were performed using ground and high altitude measurements [22]-[25]. In order to be consistent with the part using the californium source, the ground was alternatively considered as HDPE and concrete.

The geometry of the simulated scene in GEANT4 consists in a series of rectangular shapes nested in one another, including the particle source, the material block definition and the atmosphere defined as the air. As shown in Fig. 8, the volume "World" consists of vacuum is a cube whose edge is $200 \mathrm{~m}$. A smaller cube of $180 \times 180 \times 180 \mathrm{~m}^{3}$ is the volume of the atmosphere filled with air. The material block is defined by a parallelepiped with a square face of $170 \times 170 \mathrm{~m}^{2}$ and 80 $\mathrm{m}$ deep. The scorer for the albedo neutron is located $1 \mathrm{~m}$ above the material block. ATMORAD and GEANT4 simulations assess the shower spectra and the albedo neutron spectrum, respectively. 


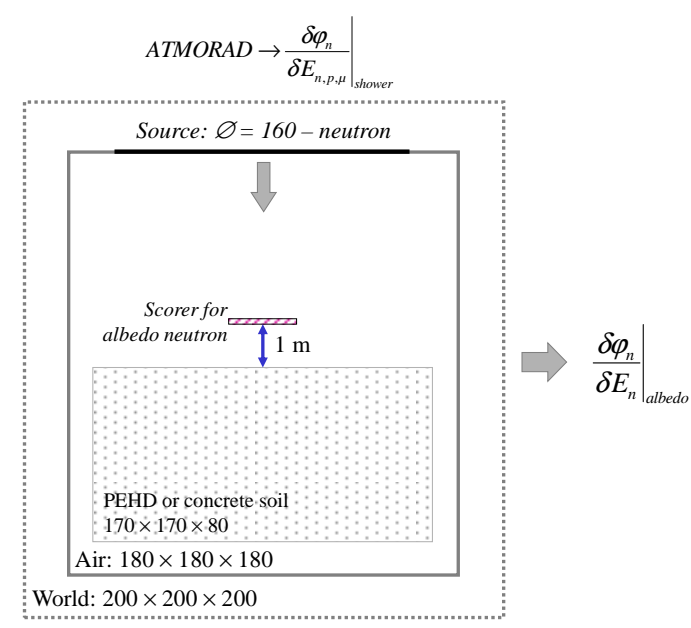

Fig. 8 Description of the modeled scene from GEANT4, the elementary volumes representing the atmosphere and the material block are represented. The particle generation disc is also visible at the top of the atmosphere (dimensions are in meter). Air shower neutron, proton and muon spectra are issued from ATMORAD calculations.

Fig. 9 presents the neutron spectra representative to Paris city obtained for three configurations: 1) only shower, 2) shower and HDPE soil, 3) shower and concrete soil.

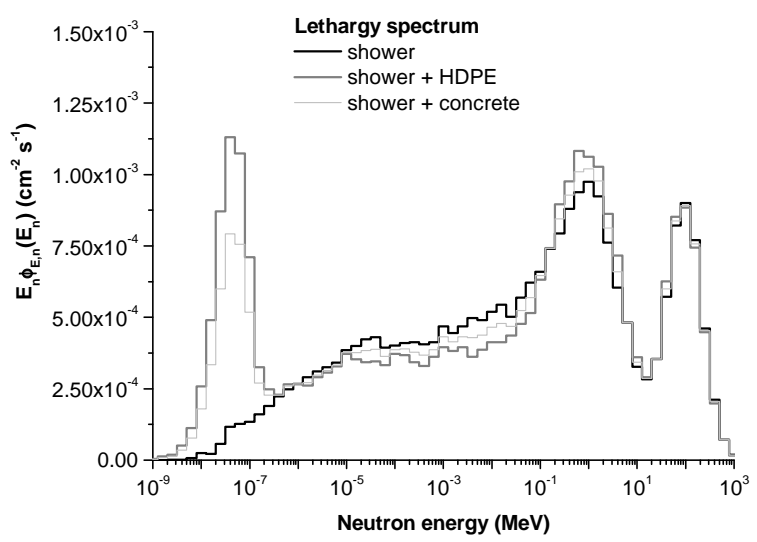

Fig. 9 Neutron spectra in Paris (48 $51^{\prime} 12^{\prime \prime} \mathrm{N}, 2^{\circ} 20^{\prime} 55^{\prime \prime} \mathrm{E}$ and $+42 \mathrm{~m}$ ) calculated using ATMORAD, considering configurations defined by: 1) only shower, 2) shower + HDPE soil and 3) shower + concrete soil

Cascade neutrons are weakly impacted by the soil modeling. This property is commonly used in cosmic ray monitoring and analyses. Moreover, evaporation neutrons are reinforced by the albedo contribution in the energy range from 0.1 to $5 \mathrm{MeV}$, i.e. a field of interest of SEU in nanoscale devices. At the same time, there is a significant impact in the thermal and epithermal domains. Indeed, a decrease in the epithermal neutron domain is visible, whereas the thermal domain is considerably reinforced. This point needsto be studied in detail because of the Boron 10, although eliminated from the point of view of the BPSG layers, which remains a chemical element present in the device for doping.

Fig. 10 presents the SER increases (in \%) as function of the technological critical charge. Two ratios are considered, firstly between the shower + HDPE and shower only, and secondly between the shower + concrete and shower only. Moreover, a perimeter is drawn to designate the typical critical charge values for the 45-nm technology. The impact of albedo neutrons is of the order of 10 to $20 \%$.

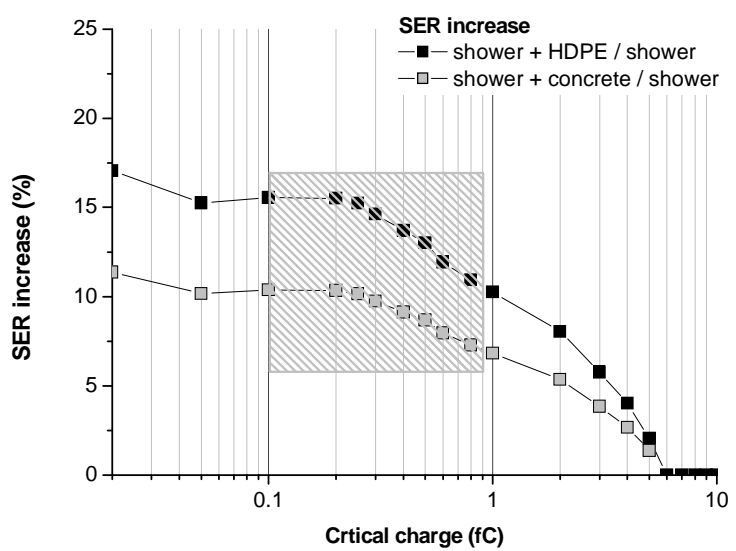

Fig. 10 SER increases (in \%) as function of the technological critical charge. Ratios between 1) shower + HDPE and shower and 2) shower + concrete and shower are presented. Perimeter designates a relevant area of typical critical charge values for the 45-nm technological node.

With continued advancements in the technological integration, the SEE sensitivity is expected to increase and experimental studies have demonstrated the occurrence due to direct ionization of protons [25]-[29] and muons [17] for nanoscale devices [18]. Moreover, it is essential to take into account the alpha emission contribution when considering terrestrial environments. The emission rate depends on the quantity and the purification grade of alpha-emitting impurities naturally present in packaging materials and materials used in the chip fabrication process. It is useful to compare the neutron albedo contribution with other contributions induced by protons, muons and alpha emitters. This work is performed by simulations using MUSCA SEP3.The $\alpha$-emitter contamination is considered as a uniform alpha-emissivity rate of $1 \times 10^{-4} \alpha / \mathrm{cm}^{2} / \mathrm{hr}$ (hyper-low-alpha, HLA [30]-[32]). Based on the same method as for neutron spectra, the muon and proton spectra are issued from ATMORAD (Paris location).

Fig. 11 presents the stacked SER contributions induced by neutron (shower and albedo), protons, muons and alpha emitters. Results are presented for three critical charge values from 0.1 to $0.4 \mathrm{fC}$.

It is complex to interpret results because threshold phenomena intervene on the SEU contributions induced by each particle type (i.e. sensitivity to protons, muons and low energy neutrons). Moreover, it is undeniable that the technology, the process and the using conditions have a significant impact on results. Concerning the 45-nm technology we investigate in this work, $\alpha$-SER is the main contribution, whatever the SEU criteria (i.e. critical charge). As the critical charge value decreases, the contributions of 
other particles increase. Neutron albedo contributions are not preponderant, they remain quite significant and it justifies their being taken into account in the terrestrial radiation field specifications.

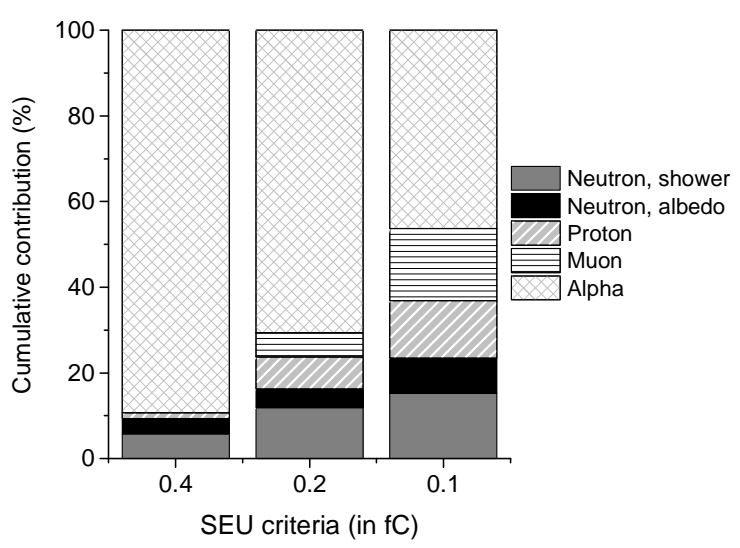

Fig. 11 Stacked contributions to the total SER as function of the SEU criteria (critical charge in fC).

Recent publications [33][34] were devoted to assess the soft error rate on advanced technologies in the field of terrestrial and/or aeronautical environments. In a study focusing on the impact of alphas emitters in $7 \mathrm{~nm}$ FinFET SRAMs, Fang et al. [34] show that alpha and neutron SER trends are similar when the voltage is low enough. The interpretation of results is based on the comparison of critical charges and the low LET alpha particle, this argument that can be extended to muons. These results seem contradictory with Fig. 11 since the trends between neutron and alpha contributions are opposite. However, impacts of technological details, integration or usage parameters (as power supply) affect drastically the SEU sensitivity. This is mainly due to threshold phenomena that can change trends over several decades. The work proposed by Trippe et al. [33] shows that muon contribution to the total SEU rate is considerably lower than the neutron contribution in 28-nm SRAMs. This trend is validated whatever the supply voltage value. This result is compatible with Fig. 11 for critical charge levels greater than $0.25 \mathrm{fC}$, for which the muon contribution is negligible. These works reveal the complexity of generalizing trends and analyzes from a given technology.

\section{CONCLUSION}

This work investigated impacts of ground albedo neutrons on the SER on a 45-nm technology, using a ${ }^{252} \mathrm{Cf}$ facility whose neutron energy range is particularly suitable for this study. Albedo neutrons are due to the soil inducing backscattered neutrons, which implies the importance of the soil properties (chemical composition, density, hydrogen content).

Thus, SER increase was recorded using a ${ }^{252} \mathrm{Cf}$ source by planning several scenarios. The first scenario considers the DUT in an environment composed of air. The two following scenarios consist in placing a material behind the DUT whose composition is alternately HDPE and concrete. Results show the impact in the neutron spectrum concerns mainly energies below to $5 \mathrm{MeV}$.

The average SER measured during the campaigns using material blocks demonstrate SER increases in comparison to the reference SER (air environment). Moreover, crosscomparisons show the importance of the material properties in the SER increases. In the case of realistic terrestrial environment, i.e. considering a neutron spectrum, the impact of albedo neutrons is of the order of 10 to $20 \%$ for HDPE and concrete material. This order of magnitude is significant and it may depend on the technological sensitivity.

This problem can be extended to the specification of radiation fields for terrestrial applications. Indeed, the impacted energy ranges, of the order of a few tens of $\mathrm{MeV}$ to a few $\mathrm{MeV}$, correspond to the sensitivity thresholds of nanoscale technologies. Moreover, the integration involves improving the radiation field assessment, especially in the evaporation energy domain. This also concerns thermal neutrons, although the Boron 10 was eliminated from the point of view of the BPSG layers. An important perspective would be to plan measurement campaigns in an adequate environment (high altitude platform), using advanced technologies and investigating the soil property impacts.

Although this work concerns the natural environment, it would be interesting to extend this analysis to accelerated neuron testing. Thus, future work will be devoted to consider various neutron facilities (LANL, RCNP, TRIUMF, MIRAGE, GENEPI etc.) and to model characteristics (fluxes and spectrum) of albedo neutron in their device test chambers.

\section{REFERENCES}

[1] G. Hubert, R. Velazco, C. Frederico, A. Cheminet, C. SilvaCardenas, L. V. E. Caldas, F. Pancher, V. Lacoste, F. Palumbo, W. Mansour, L. Artola, F. Pineda and S. Duzellier, "Continuous high-altitude measurements of cosmic ray neutron and SEU/MCU at various locations: correlation and analyses basedon MUSCA-SEP3", IEEE Trans. Nucl. Sci., Vol. 60, No. 4, pp. 2418-2426, Aug. 2013. DOI: 10.1109/TNS.2013.2240697.

[2] G. Hubert, L. Artola and D. Regis, "Impact of scaling on the soft error sensitivity of bulk, FDSOI and FinFET technologies due to atmospheric radiation", Integration, the VLSI journal, Vol. 50, pp. 39-47, June 2015. DOI: 10.1016/j.vlsi.2015.01.003.

[3] J. A. Simpson, "Neutrons Produced in the Atmosphere by the Cosmic Radiation", Phys. Rev, Vol. 83, pp. 1175-1188, 1951. DOI: 10.1103/PhysRev.83.1175.

[4] M. Zreda, "Land-surface hydrology with cosmic-ray neutrons: principles and applications", Journal of Japan Society, Soil Physics., Vol. 132, pp. 25-30, 2016.

[5] Zreda, M., Shuttleworth, W.J., Zeng, X., Zweck, C., Desilets, D., Franz, T. and Rosolem, R., "COSMOS: the COsmic-ray Soil Moisture Observing System", Hydrol. Earth Syst. Sci., Vol. 16, pp. 4079-4099, 2012. DOI: 10.5194/hess-16-4079-2012.

[6] M. Köhli, M. Schrön, M., Zreda, U., Schmidt, P., Dietrich, S., Zacharias, "Footprint characteristics revised for field-scale soil moisture monitoring with cosmic-ray neutrons", Water Resour. Res., Vol. 51, no 7, pp. 5772-5790, 2015. DOI: 10.1002/2015WR017169.

[7] G. Hubert, "Ground Albedo Neutron impacts to Seasonal Variations of Cosmic-Ray-induced Neutron in medium Geomagnetic Latitude and Antarctica: Impacts on Soft Error 
Rate”, IEEE Trans. Nucl. Sci., Vol. 64, no 4, pp. 622-629, 2016. DOI: $10.1109 /$ TNS.2016.2614540.

[8] G. Hubert, M. T. Pazianotto, C. A. Federico, "Modeling of ground albedo neutrons to investigate seasonal cosmic rayinduced neutron variations measured at high-altitude stations", Journal of Geophysical Research, Vol.121, Issue12, pp. 186201, 2016. DOI: 10.1002/2016JA02305.

[9] G. Hubert, S. Duzellier, C. Inguimbert, C. Boatella-Polo, F. Bezerra, and R. Ecoffet, "Operational SER calculations on the SAC-C orbit using the Multi SCAles Single Event Phenomena Predictive Platform (MUSCA SEP3)", IEEE Trans. Nucl. Sci., Vol. 56, No.6, pp. 3032-3042, Dec. 2009. DOI: 10.1109/TNS.2009.2034148.

[10] I. I. Bashter, "Calculation of radiation attenuation coefficients for shielding concretes", Annals of Nuclear Energy, Volume 24, Issue 17, Pages 1389-1401, Nov. 1997. DOI: 10.1016/S03064549(97)00003-0.

[11] A. Cheminet, V. Lacoste, V. Gressier, G. Hubert, A. Martin and M. Pépino, "Characterization of the IRSN neutron multisphere spectrometer (HERMEIS) at European standard calibration fields", IOP Science Journal of Instrumentation, 2nd International workshop on Fast Neutron Detectors and Applications (FNDA), Vol. 7, article C04007, 2012. DOI: 10.1088/1748-0221/7/04/C04007.

[12] G. Hubert, "Analyses of Cosmic Ray induced-Neutron based on spectrometers operated simultaneously at mid-latitude and Antarctica high-altitude stations during quiet solar activity", Astroparticle Physics, Vol. 83, pp. 30-39, Oct. 2016. DOI: 10.1016/j.astropartphys.2016.07.002.

[13] M. Matzke, "Unfolding procedures", Radiation Protection Dosimetry, Vol. 107, No. 1-3, pp. 155-174, 2003. DOI: 10.1093/oxfordjournals.rpd.a006384.

[14] M. Matzke, "Unfolding of Pulse Height Spectra: The HEPRO Program System", Report PTB-N-19, Physicalish-TechnischeBundensantalt, Braunshweig, 1994.

[15] M. Raine, G. Hubert, P. Paillet, M. Gaillardin, A. Bournet, "Implementing realistic heavy ion track in a SEE prediction tool : Comparison between different approaches", IEEE TNS, vol. 59, no. 4, pp 950-957, Aug. 2012. DOI: 10.1109/RADECS.2011.6131346.

[16] D. Lambert, F. Desnoyers, D. Thouvenot, O. Riant, J. Galinat, B. Azaiis, T. Colladant, "Single Event Upsets Induced by a few MeV Neutrons in SRAMs and FPGAs", IEEE Radiation Effects Data Workshop, 2017. DOI: 10.1109/NSREC.2017.8115444.

[17] B. D. Sierawski, R. A. Reed, M. H. Mendenhall, R. A. Weller, R. D. Schrimpf, S. J. Wen, R. Wong, N. Tam, R. C. Baumann, "Effect of scaling on Muon-Induced Soft Errors", in Proc. IEEE Int. Rel. Phys. Symp., pp. 247-252, Apr. 2011. DOI: 10.1109/IRPS.2011.5784484

[18] Y. Q. Aguiar, L. Artola, G. Hubert, C. Meinhardt, F. L. Kastensmidt, R. A. L. Reis, "Evaluation of Radiation-Induced Soft Error in Majority Voters Designed in 7nm FinFET Technology", Elsevier Microelectronics Reliability, vol. 76-77, pp. 660-664, Sept. 2017. DOI: 10.1016/j.microrel.2017.06.077.

[19] www.itrs.net

[20] G. Hubert and A. Cheminet, "Radiation effects investigations based on atmospheric radiation model (ATMORAD) considering GEANT4 simulations of extensive air showers and solar modulation potential", Radiation Research, Vol. 184, No. 1, pp. 83-94, July 2015. DOI: 10.1667/RR14028.1.

[21] G. Hubert, C. A. Federico, M. T. Pazianotto and O. L. Gonzales, "Long and short-term Atmospheric Radiation analyses based on coupled Measurements at High Altitude remote Stations and extensive Air Shower modeling", Astroparticle Physics, Vol. 74, pp.27-36, Feb. 2016. DOI: 10.1016/j.astropartphys.2015.09.005.

[22] P. Goldhagen, M. Reginatto, T. Kniss, J.W. Wilson, R.C. Singleterry et al., "Measurements of the energy spectrum of cosmic ray induced neutrons aboard an ER-2 high-altitude airplane", Nuclear Instruments and Methods in Physics Research, Vol. 476, pp. 42-51, 2002. DOI: 10.1016/S01689002(01)01386-9.

[23] M.S. Gordon, P. Goldhagen, K.P. Rodbell, T.H. Zabel, H.H.K. Tang, J.M. Clem, P. Bailey, "Measurement of the flux and energy spectrum of cosmic-ray induced neutrons on the ground", IEEE TNS, vol. 51, no. 6, pp. 3427-3434, Dec. 2004. DOI: $10.1109 /$ TNS.2004.839134.

[24] D. Maurin, A. cheminet, L. Derome, A. Ghelfi and G. Hubert, "Neutron monitors and muon detectors for solar modulation studies: interstellar flux, yield function, and assessment of critical parameters in count rate calculations", Advances in Space Research, Vol. 55, no 1, pp. 363-389, Jan. 2015. DOI: 10.1016/j.asr.2014.06.021.

[25] A. Ghelfi, D. Maurin, A. Cheminet, L. Derome, G. Hubert and F. Melot, "Neutron monitors and muon detectors for solar modulation studies: 2. $\phi$ time series", advances in space Research, Vol. 60, no 4, pp. 833-847, Aug. 2016. DOI: 10.1016/j.asr.2016.06.027.

[26] K. P. Rodbell, D. F. Heidel, H. H. K. Tang, M. S. Gordon, P. Oldiges, C. E. Murray, "Low-energy proton-induced singleevent-upsets in $65 \mathrm{~nm}$ node, silicon-on-insulator, latches and memory cells", IEEE TNS, vol. 54, no. 6, pp. 2474-2479, Dec. 2007. DOI: 10.1109/TNS.2007.909845.

[27] D. F. Heidel, P. W. Marshall, J. A. Pellish, K. P. Rodbell, K. A. LaBel, J. R. Schwank et al., "Single-event upsets and multiplebit upsets on a $45 \mathrm{~nm}$ SOI SRAM", IEEE TNS, vol. 56, no. 6, pp. 3499-3504, Dec. 2009. DOI: 10.1109/TNS.2009.2033796.

[28] B. D. Sierawski, J. A. Pellish, R. A. Reed, R. D. Schrimpf, K. M. Warren et al., "Impact of low-energy proton induced upsets on test methods and rate predictions", IEEE TNS, vol. 56, no. 6, pp. 3085-3092, Dec. 2009. DOI: 10.1109/TNS.2009.2032545.

[29] G. Hubert, S. Duzellier, F. Bezzera, R. Ecoffet, "MUSCA SEP3 contributions to investigate the direct ionization proton upset in $65 \mathrm{~nm}$ technology for space and atmospheric applications", Radiation and Its Effects on Components and Systems, Proceedings of the European Conference on Radiation and its Effects on Components and Systems (RADECS), pp. 179-186, 2009. DOI: 10.1109/RADECS.2009.5994577.

[30] R. Heald, "How Cosmic Rays Cause Computer Downtime", IEEE Rel. Soc. SCV Meeting.

[31] J.F. Ziegler and H. Puchner, "SER-History, Trends and Challenges", Cypress Semiconductors, 2004.

[32] R. Baumann and E. Smith, "Call for improved ultra-low background alpha-particle emission metrology for the semiconductor industry", Int. SEMATECH Technology Transfer, 2001

[33] J. M. Trippe, R.A. Reed, R.A. Austin, B.D. Sierawski, L.W. Massengill, R.A. Weller, K.M. Warren, R.D. Schrimpf, B. Narasimham, B. Bartz, and D. Reed, "Predicting Muon-Induced SEU Rates for a 28 -nm SRAM Using Protons and Heavy Ions to Calibrate the Sensitive Volume Model", IEEE TNS, vol.. 65, no. 2, pp. 712-718, Feb. 2018. DOI: 10.1109/TNS.2017.2786585.

[34] Y. P. Fang and A. S. Oates, "Soft Errors in 7nm FinFET SRAMs with Integrated Fan-out Packaging", IEEE International Reliability Physics Symposium (IRPS), 2018. DOI: 10.1109/IRPS.2018.8353584. 